\title{
A Polar Material with Facility of Crystal Growth and Large Second-harmonic Generation Response
}

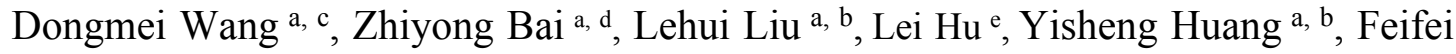
Yuan ${ }^{\mathrm{a}, \mathrm{b}}$, Dongshan Wei ${ }^{\mathrm{f}}$, Zhoubin Lin a, b,*, Lizhen Zhang a, b,*

${ }^{a}$ Key Laboratory of Optoelectronic Materials Chemistry and Physics, Fujian Institute of Research on the Structure of Matter, Chinese Academy of Sciences, Fuzhou, Fujian, 350002, P. R. China

${ }^{\mathrm{b}}$ Fujian Science \& Technology Innovation Laboratory for Optoelectronic Information of China, Fuzhou, Fujian, 350108, China

c College of Chemistry and Materials Science, Fujian Normal University, Fuzhou ,350007, China

${ }^{d}$ University of Chinese Academy of Sciences, Beijing 100049, P. R. China

e School of Environmental and Chemical Engineering, Chongqing Three Gorges University, Chongqing, 404100, China

${ }^{\mathrm{f}}$ School of Electronic Engineering, Dongguan University of Technology, Dongguan, 523808, China

*Corresponding author, E-mail: lzb@fjirsm.ac.cn; lzzhang@fjirsm.ac.cn. 
Table S1 Fractional Atomic Coordinates $\left(\times 10^{4}\right)$ and Equivalent Isotropic Displacement Parameters $\left(\AA^{2} \times 10^{3}\right)$ for AHIO. $U_{\text {eq }}$ is defined as $1 / 3$ of of the trace of the orthogonalised $\mathrm{U}_{\mathrm{IJ}}$ tensor.

\begin{tabular}{|c|c|c|c|c|}
\hline \multicolumn{2}{|c|}{ Atom $x$} & \multirow{2}{*}{$\begin{array}{l}\mathrm{y} \\
12964.7(3)\end{array}$} & \multirow{2}{*}{$\begin{array}{l}z \\
6513.5(4)\end{array}$} & \multirow{2}{*}{$\begin{array}{l}\mathrm{U}(\mathrm{eq}) \\
12.45(10)\end{array}$} \\
\hline $\mathbf{I}_{1}$ & $3344.0(2)$ & & & \\
\hline $\mathbf{I}_{2}$ & 2760.1(3) & 11649.1(3) & $3905.8(3)$ & $12.48(10)$ \\
\hline $\mathbf{I}_{3}$ & $4460.3(3)$ & 9463.3(3) & 8868.8(4) & $14.75(11)$ \\
\hline $\mathbf{I}_{4}$ & 3391.1(3) & $10202.6(3)$ & $6211.5(5)$ & $19.38(11)$ \\
\hline $\mathbf{I}_{5}$ & $1657.1(3)$ & $7664.7(3)$ & $4210.5(4)$ & $13.67(11)$ \\
\hline $\mathbf{A l}_{1}$ & $\mathbf{0}$ & 10000 & $1694(3)$ & 11.3(7) \\
\hline $\mathbf{A l}_{2}$ & 3333.33 & 6666.67 & $6686(3)$ & 13.1(7) \\
\hline $\mathbf{A l}_{3}$ & 3333.33 & 6666.67 & $1679(3)$ & $12.2(7)$ \\
\hline $\mathbf{O}_{1}$ & $4325(3)$ & $13280(4)$ & $5576(4)$ & 19.2(11) \\
\hline $\mathbf{O}_{2}$ & $3416(4)$ & $12074(4)$ & $7299(5)$ & 30.5(13) \\
\hline $\mathbf{O}_{3}$ & $2369(3)$ & 12191(3) & 5588(4) & $18.6(11)$ \\
\hline $\mathbf{O}_{4}$ & $3042(4)$ & $11214(3)$ & 2111(4) & 23.3(11) \\
\hline $\mathbf{O}_{5}$ & $2220(3)$ & $12259(3)$ & 3248(4) & $18.7(11)$ \\
\hline $\mathbf{O}_{6}$ & $1700(3)$ & $10529(3)$ & $4173(4)$ & $18.0(10)$ \\
\hline $\mathbf{O}_{7}$ & $656(3)$ & 9551(3) & $2536(4)$ & $16.4(10)$ \\
\hline $\mathbf{O}_{8}$ & $-463(3)$ & 8889(3) & $850(4)$ & $16.9(10)$ \\
\hline $\mathbf{O}_{9}$ & $3516(4)$ & $10798(4)$ & 5013(5) & 40.1(16) \\
\hline $\mathbf{O}_{10}$ & 2387(3) & $9037(4)$ & $5980(5)$ & 28.1(13) \\
\hline $\mathbf{O}_{11}$ & $4400(4)$ & 9985(4) & 5993(5) & $29.6(13)$ \\
\hline $\mathbf{O}_{12}$ & $5227(4)$ & $9832(4)$ & $7677(4)$ & 23.4(12) \\
\hline $\mathbf{O}_{13}$ & 3329(3) & 8997(3) & $8150(4)$ & $18.3(10)$ \\
\hline $\mathbf{O}_{14}$ & $4422(3)$ & 8347(3) & $9132(4)$ & $19.5(11)$ \\
\hline $\mathbf{O}_{15}$ & $3832(3)$ & 7784(3) & $5860(4)$ & 17.4(11) \\
\hline $\mathbf{O}_{16}$ & $2886(3)$ & 7933(3) & 4212(4) & $18.5(10)$ \\
\hline $\mathbf{O}_{17}$ & $1216(4)$ & $6919(4)$ & $5400(5)$ & 23.1(12) \\
\hline $\mathbf{O}_{18}$ & $1173(3)$ & $6705(3)$ & $3230(4)$ & 19.4(11) \\
\hline $\mathbf{O}_{19}$ & 3754(3) & $7776(3)$ & 2521(4) & $15.6(10)$ \\
\hline $\mathbf{O}_{20}$ & 2922(3) & 5558(3) & $840(4)$ & $17.0(10)$ \\
\hline $\mathbf{O}_{21}$ & 4448(3) & $7297(3)$ & $7533(4)$ & $16.5(10)$ \\
\hline
\end{tabular}


Table S2 Anisotropic Displacement Parameters $\left(\AA^{\left.2 \times 10^{3}\right)}\right.$ for AHIO. The Anisotropic displacement factor exponent takes the form: $-2 \pi^{2}\left[h^{2} a * 2 U_{11}+2 h k a * b * U_{12}+\ldots\right]$.

\begin{tabular}{|c|c|c|c|c|c|c|}
\hline \multicolumn{2}{|c|}{ Atom $U_{11}$} & \multirow{2}{*}{$\begin{array}{l}U_{22} \\
12.21(17)\end{array}$} & \multirow{2}{*}{$\begin{array}{l}U_{33} \\
13.7(2)\end{array}$} & \multirow{2}{*}{$\begin{array}{l}U_{23} \\
-0.83(17)\end{array}$} & \multirow{2}{*}{$\begin{array}{l}U_{13} \\
-0.65(16)\end{array}$} & \multirow{2}{*}{$\begin{array}{l}U_{12} \\
4.91(14)\end{array}$} \\
\hline$I_{1}$ & $10.40(17)$ & & & & & \\
\hline $\mathbf{I}_{2}$ & $10.03(17)$ & $10.09(17)$ & $16.1(2)$ & $1.55(16)$ & $0.18(16)$ & $4.09(14)$ \\
\hline $\mathbf{I}_{3}$ & $14.37(18)$ & $12.02(18)$ & $18.3(3)$ & $-0.81(17)$ & $-3.05(17)$ & $6.94(15)$ \\
\hline $\mathbf{I}_{4}$ & $13.97(19)$ & $19.9(2)$ & 23.1(3) & $0.0(2)$ & $-0.21(18)$ & $7.54(16)$ \\
\hline $\mathbf{I}_{5}$ & $10.39(17)$ & $13.40(18)$ & $18.8(3)$ & $-0.29(17)$ & $0.16(16)$ & $7.12(15)$ \\
\hline $\mathbf{A l}_{1}$ & $9.3(8)$ & $9.3(8)$ & $15.2(19)$ & $\mathbf{0}$ & $\mathbf{0}$ & $4.7(4)$ \\
\hline $\mathbf{A l}_{2}$ & 10.4(9) & $10.4(9)$ & $18(2)$ & $\mathbf{0}$ & $\mathbf{0}$ & $5.2(4)$ \\
\hline $\mathbf{A l}_{3}$ & 11.4(9) & $11.4(9)$ & $14(2)$ & $\mathbf{0}$ & $\mathbf{0}$ & $5.7(4)$ \\
\hline $\mathbf{O}_{1}$ & $16(2)$ & $21(2)$ & $23(3)$ & $4(2)$ & $5(2)$ & 11(2) \\
\hline $\mathbf{O}_{2}$ & $37(3)$ & $25(3)$ & $33(4)$ & $9(3)$ & $-1(3)$ & $18(3)$ \\
\hline $\mathbf{O}_{3}$ & $11(2)$ & $20(2)$ & 19(3) & $-7(2)$ & $-1.3(19)$ & $2.9(19)$ \\
\hline $\mathbf{O}_{4}$ & 33(3) & 19(2) & $20(3)$ & $-1(2)$ & $6(2)$ & $14(2)$ \\
\hline $\mathrm{O}_{5}$ & $17(2)$ & $15(2)$ & 21(3) & $1(2)$ & $-3(2)$ & $9(2)$ \\
\hline $\mathbf{O}_{6}$ & $13(2)$ & $14(2)$ & $17(3)$ & $0(2)$ & $-1(2)$ & $-1.5(18)$ \\
\hline $\mathbf{O}_{7}$ & $16(2)$ & $12(2)$ & $21(3)$ & $2(2)$ & $-2(2)$ & $6.8(19)$ \\
\hline $\mathbf{O}_{8}$ & $10(2)$ & $16(2)$ & $20(3)$ & $-6(2)$ & $1.6(19)$ & $2.2(19)$ \\
\hline $\mathbf{O}_{9}$ & $34(3)$ & $46(4)$ & 44(4) & $27(3)$ & $6(3)$ & $22(3)$ \\
\hline $\mathbf{O}_{10}$ & $18(2)$ & 21(3) & 29(3) & $-7(2)$ & $1(2)$ & $2(2)$ \\
\hline $\mathbf{O}_{11}$ & $27(3)$ & $45(3)$ & $25(3)$ & $10(3)$ & $4(2)$ & $24(3)$ \\
\hline $\mathbf{O}_{12}$ & $17(2)$ & $36(3)$ & 21(3) & $5(2)$ & $4(2)$ & $17(2)$ \\
\hline $\mathbf{O}_{13}$ & $14(2)$ & $17(2)$ & 21(3) & $-1(2)$ & $-5(2)$ & $9.1(19)$ \\
\hline $\mathbf{O}_{14}$ & 31(3) & $19(2)$ & $15(3)$ & $-2(2)$ & $-4(2)$ & $17(2)$ \\
\hline $\mathbf{O}_{15}$ & $13(2)$ & $15(2)$ & $23(3)$ & $5(2)$ & $-1(2)$ & $5.2(19)$ \\
\hline $\mathbf{O}_{16}$ & $10(2)$ & $33(3)$ & $15(3)$ & $-2(2)$ & $-0.9(19)$ & $12.5(19)$ \\
\hline $\mathbf{O}_{17}$ & $23(3)$ & 29(3) & $23(3)$ & 11(2) & $12(2)$ & $18(2)$ \\
\hline $\mathbf{O}_{18}$ & $18(2)$ & $17(2)$ & $24(3)$ & $-5(2)$ & $-3(2)$ & $9(2)$ \\
\hline $\mathbf{O}_{19}$ & $9(2)$ & $14(2)$ & 21(3) & $-6(2)$ & $-1(2)$ & 3.9(18) \\
\hline $\mathbf{O}_{20}$ & $15(2)$ & $13(2)$ & $19(3)$ & $-5(2)$ & $2(2)$ & 3.3(19) \\
\hline $\mathbf{O}_{21}$ & $15(2)$ & $17(2)$ & $20(3)$ & $-4(2)$ & $-3(2)$ & $10(2)$ \\
\hline
\end{tabular}


Table S3 Bond Lengths for AHIO.

\begin{tabular}{|c|c|c|c|c|c|}
\hline \multicolumn{3}{|c|}{ Atom Atom Length/Å } & \multicolumn{3}{|c|}{ Atom Atom Length $/ \AA ̊$} \\
\hline$\overline{I_{1}}$ & $\mathbf{O}_{3}$ & $1.839(5)$ & $\mathbf{A l}_{1}$ & $\mathrm{O}_{8}{ }^{1}$ & $1.877(5)$ \\
\hline $\mathbf{I}_{1}$ & $\mathbf{O}_{1}$ & $1.819(5)$ & $\mathbf{A l}_{1}$ & $\mathbf{O}_{8}^{2}$ & $1.877(5)$ \\
\hline $\mathbf{I}_{1}$ & $\mathbf{O}_{2}$ & $1.785(5)$ & $\mathbf{A l}_{1}$ & $\mathbf{O}_{8}$ & $1.877(5)$ \\
\hline $\mathbf{I}_{2}$ & $\mathbf{O}_{6}$ & $1.791(4)$ & $\mathbf{A l}_{1}$ & $\mathbf{O}_{7}$ & $1.871(5)$ \\
\hline $\mathbf{I}_{2}$ & $\mathbf{O}_{4}$ & $1.897(5)$ & $\mathbf{A l}_{2}$ & $\mathrm{O}_{21}^{4}$ & $1.881(5)$ \\
\hline $\mathbf{I}_{2}$ & $\mathbf{O}_{5}$ & $1.801(5)$ & $\mathbf{A l}_{2}$ & $\mathrm{O}_{21}{ }^{3}$ & $1.881(5)$ \\
\hline $\mathbf{I}_{3}$ & $\mathbf{O}_{13}$ & $1.821(5)$ & $\mathbf{A l}_{2}$ & $\mathbf{O}_{21}$ & $1.881(5)$ \\
\hline $\mathbf{I}_{3}$ & $\mathbf{O}_{12}$ & $1.825(5)$ & $\mathbf{A l}_{2}$ & $\mathbf{O}_{15}$ & $1.868(5)$ \\
\hline $\mathbf{I}_{3}$ & $\mathbf{O}_{14}$ & $1.799(4)$ & $\mathbf{A l}_{2}$ & $\mathbf{O}_{15^{3}}$ & $1.868(5)$ \\
\hline $\mathbf{I}_{4}$ & $\mathbf{O}_{11}$ & $1.857(5)$ & $\mathbf{A l}_{2}$ & $\mathbf{O}_{15}^{4}$ & $1.868(5)$ \\
\hline $\mathbf{I}_{4}$ & $\mathbf{O}_{10}$ & $1.797(5)$ & $\mathbf{A l}_{3}$ & $\mathbf{O}_{19}^{3}$ & $1.880(5)$ \\
\hline $\mathbf{I}_{4}$ & $\mathbf{O}_{9}$ & $1.779(6)$ & $\mathbf{A l}_{3}$ & $\mathrm{O}_{19}^{4}$ & $1.880(5)$ \\
\hline $\mathbf{I}_{5}$ & $\mathbf{O}_{17}$ & $1.808(5)$ & $\mathbf{A l}_{3}$ & $\mathbf{O}_{19}$ & $1.880(5)$ \\
\hline $\mathbf{I}_{5}$ & $\mathbf{O}_{18}$ & $1.809(5)$ & $\mathbf{A l}_{3}$ & $\mathbf{O}_{20}$ & $1.879(5)$ \\
\hline $\mathbf{I}_{5}$ & $\mathbf{O}_{16}$ & $1.804(4)$ & $\mathbf{A l}_{3}$ & $\mathbf{O}_{20}^{3}$ & $1.879(5)$ \\
\hline $\mathbf{A l}_{1}$ & $\mathbf{O}_{7}^{1}$ & $1.871(5)$ & $\mathbf{A l}_{3}$ & $\mathbf{O}_{20}{ }^{4}$ & $1.879(5)$ \\
\hline $\mathbf{A l}_{1}$ & $\mathbf{O}_{7}^{2}$ & $1.871(5)$ & & & \\
\hline
\end{tabular}


Table S4 Bond Angles for AHIO.

\begin{tabular}{|c|c|c|c|c|c|c|}
\hline \multicolumn{4}{|c|}{ Atom Atom Atom Angle ${ }^{\circ}$} & \multicolumn{3}{|c|}{ Atom Atom Atom Angle $/^{\circ}$} \\
\hline$\overline{O_{1}}$ & $\mathbf{I}_{1}$ & $\mathbf{O}_{3}$ & $96.9(2)$ & $\mathrm{O}_{21}{ }^{3} \quad \mathrm{Al}_{2}$ & $\mathrm{O}_{21}{ }^{4}$ & 91.9(3) \\
\hline $\mathbf{O}_{2}$ & $\mathbf{I}_{1}$ & $\mathbf{O}_{3}$ & $99.9(2)$ & $\mathbf{O}_{21}{ }^{3} \quad \mathrm{Al}_{2}$ & $\mathbf{O}_{21}$ & 91.9(3) \\
\hline $\mathbf{O}_{2}$ & $\mathbf{I}_{1}$ & $\mathbf{O}_{1}$ & $100.2(2)$ & $\mathbf{O}_{15} \quad \mathrm{Al}_{2}$ & $\mathbf{O}_{21}{ }^{4}$ & $179.0(3)$ \\
\hline $\mathbf{O}_{5}$ & $\mathbf{I}_{2}$ & $\mathbf{O}_{3}$ & 84.9(2) & $\mathbf{O}_{15} \quad \mathbf{A l}_{2}$ & $\mathbf{O}_{21}{ }^{3}$ & 88.1(2) \\
\hline $\mathbf{O}_{5}$ & $\mathbf{I}_{2}$ & $\mathbf{O}_{4}$ & $95.2(2)$ & $\mathrm{O}_{15}^{4} \quad \mathrm{Al}_{2}$ & $\mathrm{O}_{21}{ }^{4}$ & $87.2(2)$ \\
\hline $\mathbf{O}_{6}$ & $\mathbf{I}_{2}$ & $\mathbf{O}_{3}$ & 85.19(19) & $\mathbf{O}_{15}{ }^{3} \quad \mathrm{Al}_{2}$ & $\mathbf{O}_{21}{ }^{4}$ & 88.1(2) \\
\hline $\mathbf{O}_{6}$ & $\mathbf{I}_{2}$ & $\mathbf{O}_{5}$ & $99.0(2)$ & $\begin{array}{ll}\mathbf{O}_{15} & \mathbf{A l}_{2}\end{array}$ & $\mathbf{O}_{21}$ & 87.2(2) \\
\hline $\mathbf{O}_{6}$ & $\mathbf{I}_{2}$ & $\mathbf{O}_{4}$ & 93.9(2) & $\mathrm{O}_{15}^{4} \quad \mathrm{Al}_{2}$ & $\mathbf{O}_{21}{ }^{3}$ & 179.0(3) \\
\hline $\mathbf{O}_{4}$ & $\mathbf{I}_{2}$ & $\mathbf{O}_{3}$ & 179.1(2) & $\mathbf{O}_{15}{ }^{3} \quad \mathrm{Al}_{2}$ & $\mathbf{O}_{21}$ & $179.0(3)$ \\
\hline $\mathbf{O}_{13}$ & $\mathbf{I}_{3}$ & $\mathbf{O}_{12}$ & $96.5(2)$ & $\mathbf{O}_{15}{ }^{3} \quad \mathrm{Al}_{2}$ & $\mathbf{O}_{21}{ }^{3}$ & $87.2(2)$ \\
\hline $\mathbf{O}_{14}$ & $\mathbf{I}_{3}$ & $\mathbf{O}_{13}$ & $98.6(2)$ & $\mathbf{O}_{15}^{4} \quad \mathrm{Al}_{2}$ & $\mathbf{O}_{21}$ & 88.1(2) \\
\hline $\mathbf{O}_{14}$ & $\mathbf{I}_{3}$ & $\mathbf{O}_{12}$ & $98.7(2)$ & $\mathbf{O}_{15} \quad \mathbf{A l}_{2}$ & $\mathbf{O}_{15}{ }^{4}$ & 92.8(3) \\
\hline $\mathbf{O}_{10}$ & $\mathbf{I}_{4}$ & $\mathbf{O}_{11}$ & $100.6(2)$ & $\mathbf{O}_{15}{ }^{3} \quad \mathrm{Al}_{2}$ & $\mathbf{O}_{15}{ }^{4}$ & $92.8(3)$ \\
\hline $\mathbf{O}_{9}$ & $\mathbf{I}_{4}$ & $\mathbf{O}_{11}$ & 94.1(2) & $\mathbf{O}_{15} \quad \mathrm{Al}_{2}$ & $\mathbf{O}_{15^{3}}$ & 92.8(3) \\
\hline $\mathbf{O}_{9}$ & $\mathbf{I}_{4}$ & $\mathbf{O}_{10}$ & $101.2(3)$ & $\mathrm{O}_{19}{ }^{3} \quad \mathrm{Al}_{3}$ & $\mathbf{O}_{19}$ & 92.1(3) \\
\hline $\mathbf{O}_{17}$ & $\mathbf{I}_{5}$ & $\mathbf{O}_{18}$ & $97.0(2)$ & $\mathrm{O}_{20}^{4} \quad \mathrm{Al}_{3}$ & $\mathbf{O}_{19}$ & 88.1(2) \\
\hline $\mathbf{O}_{16}$ & $\mathbf{I}_{5}$ & $\mathbf{O}_{17}$ & $100.3(2)$ & $\begin{array}{ll}\mathbf{O}_{20} & \mathbf{A l}_{3}\end{array}$ & $\mathrm{O}_{19}{ }^{4}$ & $87.5(2)$ \\
\hline $\mathbf{O}_{16}$ & $\mathbf{I}_{5}$ & $\mathbf{O}_{18}$ & $99.1(2)$ & $\begin{array}{ll}\mathbf{O}_{20}{ }^{4} & \mathrm{Al}_{3}\end{array}$ & $\mathbf{O}_{19}{ }^{4}$ & $179.5(2)$ \\
\hline $\mathbf{O}_{7}{ }^{1}$ & $\mathbf{A l}_{1}$ & $\mathbf{O}_{7}^{2}$ & 91.9(3) & $\mathbf{O}_{20}{ }^{3} \quad \mathrm{Al}_{3}$ & $O_{19}$ & $87.5(2)$ \\
\hline $\mathbf{O}_{7}{ }^{1}$ & $\mathbf{A l}_{1}$ & $\mathbf{O}_{7}$ & 91.9(3) & $\mathbf{O}_{20}{ }^{3} \quad \mathrm{Al}_{3}$ & $\mathrm{O}_{19}{ }^{4}$ & 88.1(2) \\
\hline $\mathbf{O}_{7}$ & $\mathbf{A l}_{1}$ & $\mathbf{O}_{7}^{2}$ & 91.9(3) & $\begin{array}{ll}\mathbf{O}_{20} & \mathbf{A l}_{3}\end{array}$ & $\mathbf{O}_{19}$ & $179.5(2)$ \\
\hline $\mathbf{O}_{7}$ & $\mathbf{A l}_{1}$ & $\mathrm{O}_{8}{ }^{1}$ & $87.7(2)$ & $\mathbf{O}_{20}{ }^{3} \quad \mathrm{Al}_{3}$ & $\mathrm{O}_{19^{3}}$ & $179.5(2)$ \\
\hline $\mathbf{O}_{7}{ }^{1}$ & $\mathbf{A l}_{1}$ & $\mathrm{O}_{8}{ }^{1}$ & 88.5(2) & $\begin{array}{ll}\mathbf{O}_{20} & \mathbf{A l}_{3}\end{array}$ & $\mathrm{O}_{19^{3}}$ & 88.1(2) \\
\hline $\mathbf{O}_{7}{ }^{1}$ & $\mathbf{A l}_{1}$ & $\mathbf{O}_{8}$ & $179.5(2)$ & $\mathbf{O}_{20}{ }^{3} \quad \mathrm{Al}_{3}$ & $\mathbf{O}_{20}$ & $92.3(2)$ \\
\hline $\mathbf{O}_{7}$ & $\mathbf{A l}_{1}$ & $\mathbf{O}_{8}$ & $88.5(2)$ & $\mathrm{O}_{20}^{4} \quad \mathrm{Al}_{3}$ & $\mathrm{O}_{20}{ }^{3}$ & $92.3(2)$ \\
\hline $\mathbf{O}_{7^{2}}^{2}$ & $\mathbf{A l}_{1}$ & $\mathbf{O}_{8}$ & $87.7(2)$ & $\mathbf{O}_{20}{ }^{4} \quad \mathrm{Al}_{3}$ & $\mathbf{O}_{20}$ & 92.3(3) \\
\hline $\mathrm{O}_{8}{ }^{2}$ & $\mathbf{A l}_{1}$ & $\mathrm{O}_{8}{ }^{1}$ & 91.9(3) & $\mathrm{O}_{19}{ }^{3} \quad \mathrm{Al}_{3}$ & $O_{19}^{4}$ & 92.1(3) \\
\hline $\mathbf{O}_{21}$ & $\mathbf{A l}_{2}$ & $\mathrm{O}_{21}^{4}$ & 91.9(3) & $\mathbf{O}_{19} \quad \mathrm{Al}_{3}$ & $\mathrm{O}_{19}{ }^{4}$ & 92.1(3) \\
\hline
\end{tabular}


Table S5 Torsion Angles for AHIO.

\begin{tabular}{|c|c|}
\hline A BC D Angle $/^{\circ}$ & A BC D Angle $/^{\circ}$ \\
\hline $\begin{array}{llll} & \mathrm{I} & \mathrm{O} & \\
& & & \mathrm{I}_{2} \\
1 & 14.1(3)\end{array}$ & \begin{tabular}{lllll} 
& & \multicolumn{1}{l}{} & $O$ & \\
$O_{2}$ & & & $I_{2}-87.5(3)$
\end{tabular} \\
\hline
\end{tabular}

Table S6 Hydrogen Atom Coordinates $\left(\AA \times 10^{4}\right)$ and Isotropic Displacement Parameters $\left(\AA^{2} \times 10^{3}\right)$ for AHIO.

\begin{tabular}{|c|c|c|c|c|}
\hline \multicolumn{2}{|c|}{ Atom $x$} & \multirow{2}{*}{$\begin{array}{l}y \\
8960(20)\end{array}$} & \multirow{2}{*}{$\begin{array}{l}z \\
2150(60)\end{array}$} & \multirow{2}{*}{$\begin{array}{l}\mathrm{U}(\mathrm{eq}) \\
25\end{array}$} \\
\hline $\mathbf{H}_{7 \mathrm{~A}}$ & $440(50)$ & & & \\
\hline $\mathbf{H}_{7 \mathrm{~B}}$ & $1040(50)$ & $9860(40)$ & $3030(50)$ & 25 \\
\hline $\mathbf{H}_{\mathbf{8 A}}$ & $-130(40)$ & $8830(40)$ & $390(50)$ & 25 \\
\hline $\mathbf{H}_{8 \mathrm{~B}}$ & $-1030(20)$ & $8460(30)$ & $900(60)$ & 25 \\
\hline $\mathbf{H}_{11}$ & $4740(50)$ & $10060(70)$ & $6520(50)$ & 44 \\
\hline $\mathrm{H}_{12}$ & $5830(20)$ & $10130(60)$ & $7730(70)$ & 35 \\
\hline $\mathrm{H}_{15 \mathrm{~A}}$ & $3520(40)$ & $7850(40)$ & $5350(50)$ & 21 \\
\hline $\mathrm{H}_{15 \mathrm{~B}}$ & $4420(20)$ & $8180(40)$ & $5810(60)$ & 21 \\
\hline $\mathrm{H}_{19 \mathrm{~A}}$ & $3450(40)$ & $7860(40)$ & $3030(50)$ & 23 \\
\hline $\mathrm{H}_{19 \mathrm{~B}}$ & $4310(20)$ & $8160(40)$ & $2730(60)$ & 23 \\
\hline $\mathrm{H}_{20 \mathrm{~A}}$ & $3240(40)$ & $5510(40)$ & $320(50)$ & 25 \\
\hline $\mathbf{H}_{20 \mathrm{~B}}$ & $2360(30)$ & $5090(30)$ & $830(60)$ & 25 \\
\hline $\mathrm{H}_{26 \mathrm{~A}}$ & $4830(40)$ & $7100(50)$ & $7620(60)$ & 25 \\
\hline $\mathbf{H}_{26 \mathrm{~B}}$ & $4460(40)$ & $7590(50)$ & $8110(40)$ & 25 \\
\hline
\end{tabular}


Table S7. Crystal data and structure refinement for AHIO.

\begin{tabular}{|c|c|}
\hline Empirical formula & $\mathbf{A l H}_{14} \mathbf{I}_{5} \mathbf{O}_{21}$ \\
\hline Formula weight & 1011.59 \\
\hline Temperature/K & 298.76(10) \\
\hline Crystal system & hexagonal \\
\hline Space group & $\mathrm{Pb}_{3}$ \\
\hline $\mathbf{a} / \AA ̊$ & $16.1212(2)$ \\
\hline $\mathrm{c} / \AA ̊$ & $12.39760(10)$ \\
\hline Volume/Å3 & $2790.38(7)$ \\
\hline $\mathbf{Z}$ & 6 \\
\hline $\mathbf{F}(\mathbf{0 0 0})$ & 2760.0 \\
\hline Radiation & $\operatorname{Ga~K\alpha }(\lambda=1.3405)$ \\
\hline $2 \theta$ range for data collection/ ${ }^{\circ}$ & 5.504 to 121.188 \\
\hline Index ranges & $\begin{array}{l}-20 \leq \mathrm{h} \leq 20,-20 \leq \mathrm{k} \leq 19,-16 \leq \mathrm{l} \leq \\
15\end{array}$ \\
\hline Reflections collected & 36383 \\
\hline Independent reflections & $4167\left[R_{\text {int }}=0.0642, R_{\text {sigma }}=0.0324\right]$ \\
\hline Data/restraints/parameters & $4167 / 27 / 287$ \\
\hline Goodness-of-fit on $F^{2}$ & 1.012 \\
\hline Final $R$ indexes $[I>=2 \sigma(I)]$ & $R_{1}=0.0201, w_{2}=0.0466$ \\
\hline Final $R$ indexes [all data] & $R_{1}=0.0209, w R_{2}=0.0469$ \\
\hline Largest diff. peak/hole / e $\AA^{-3}$ & $0.72 /-0.92$ \\
\hline Flack parameter & $0.001(7)$ \\
\hline \multicolumn{2}{|c|}{$\begin{array}{l}\mathbf{R}_{\mathbf{1}}=\sum\left\|\mathbf{F}_{\mathbf{0}}|-| \mathbf{F}_{\mathrm{c}}\right\| / \sum\left|\mathbf{F}_{\mathbf{0}}\right| \text { for } \mathbf{F}_{\mathbf{0}}{ }^{2}>\mathbf{2 \sigma}\left(\mathbf{F}_{\mathbf{0}}{ }^{2}\right) ; \mathbf{w R}_{2}=\sum\left[\mathbf{w}\left(\mathbf{F}_{\mathbf{0}}{ }^{2}-\mathbf{F}_{\mathbf{c}}{ }^{2}\right)\right] / \\
\sum\left[\mathbf{w}\left(\mathbf{F}_{\mathbf{0}}^{2}\right)^{2}\right]^{1 / 2}\end{array}$} \\
\hline
\end{tabular}


(a)

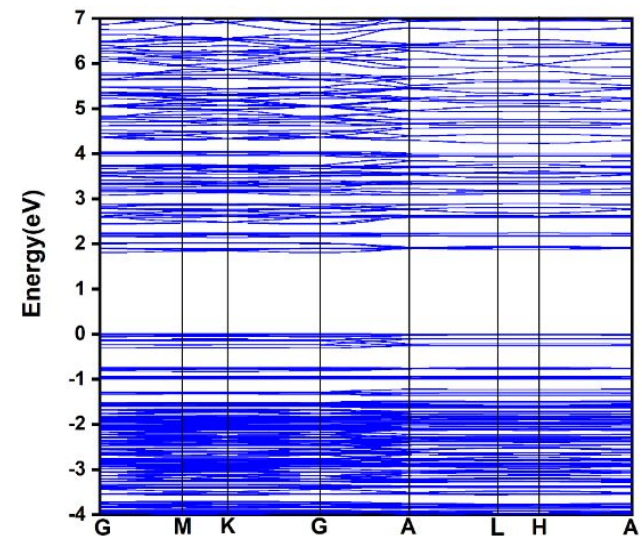

(b)

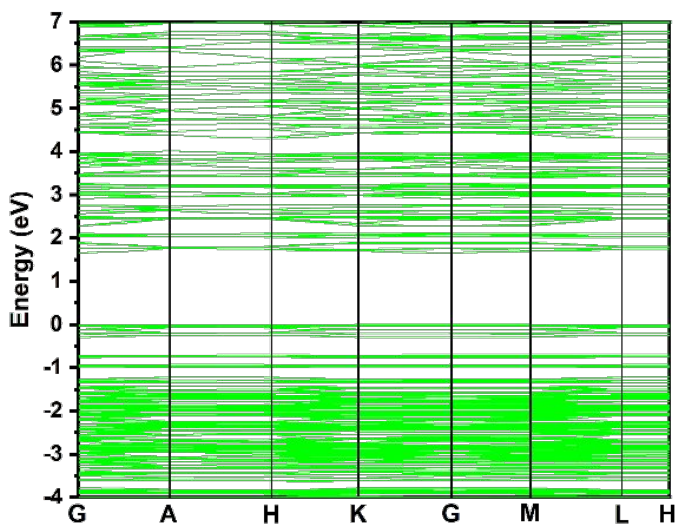

Figure S1. Calculated electronic band structure of AHIO based on VASP package (a) and CASTEP package (b).

(a)

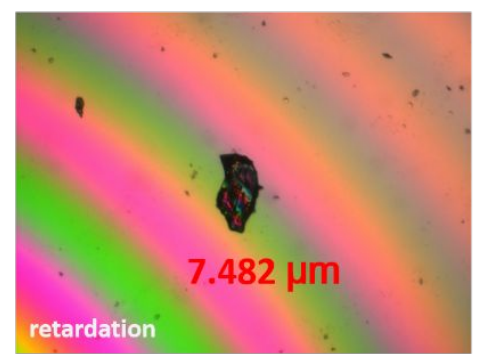

(c)

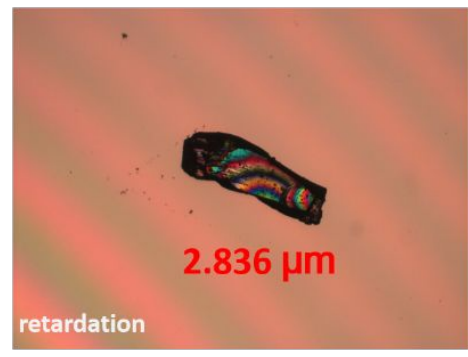

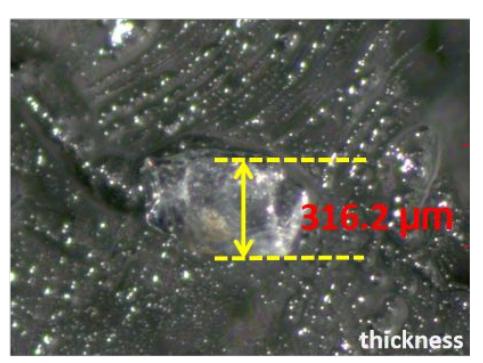

(b)

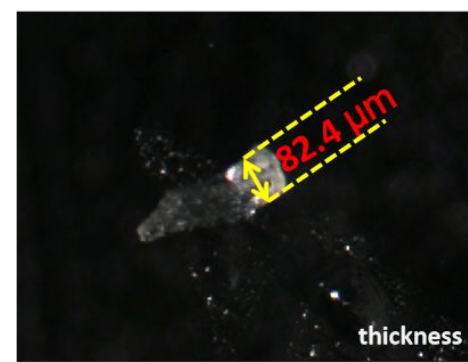

(d)

Figure S2. Birefringence measurement. (a) and (c) are the retardation for different samples while (b) and (d) are the corresponding thickness. 